\title{
Stellar formation in Hi interstellar bubbles around massive stars
}

\author{
M. C. Martín ${ }^{1}$, G. A. Romero ${ }^{1}$ and C. E. Cappa ${ }^{1,2}$ \\ ${ }^{1}$ Instituto Argentino de Radioastronomía, Argentina \\ ${ }^{2}$ Facultad de Ciencias Astronómicas y Geofisicas, UNLP, Argentina
}

Stellar winds from $\mathrm{O}$ and WR stars transfer large amounts of mechanical energy and momentum into the interstellar medium. They sweep up and compress the interstellar material, creating interstellar bubbles. These structures are detected as optical ring nebulae, as thermal radio continuum sources, as infrared shells, as neutral gas voids and expanding shells in the Hi line emission distribution, and as molecular shells.

Stellar formation may be induced in the compressed and dense regions around the ionized bubbles. Among the processes proposed for the onset of stellar formation, the "collect and collapse" process (Elmegreen \& Lada 1977) may work in these neutral structures. Observational evidence of this process includes the presence of dense and neutral gas layers surrounding the ionized regions and high density clumps within the neutral shells.

To investigate if stellar forming processes are going on in the outer neutral shells of interstellar bubbles, we analyzed the correlation of the observed neutral gas structures with stellar formation indicators. The selected regions are the neutral gas structures associated with Anon(WR 23), RCW 52, G307.27+0.27, and RCW 78 (Cappa et al. 2005a,2005b, Martín et al. 2007) linked to WR 23 (WC6), LSS 1887 (O8V), S 221 (O6), and WR 55 (WN7), respectively.

To search for young stellar objects (YSO) in the selected regions we used the 2MASS All-Sky Catalog of Point Sources (Cutri et al. 2003), the IRAS Point Source Catalogue, and the MSX6C Infrared Point Source Catalog (Egan et al. 2003).

YSO candidates were detected towards the four selected regions. Most of the YSO candidates are projected onto areas showing HI gas and some of them can be related to the neutral shells. Almost no YSO candidates were found projected onto the Hi cavities. A more complete study is necessary to verify if the scenario of "collect and collapse" is going on in the neutral shells around the nebulae.

Keywords. ISM: bubbles - ISM: individual (Anon[WR 23], RCW 52, RCW 78) - stars: formation

\section{Acknowledgements}

C.E.C. would like to thank financial support from the IAU and the Academy of Sciences of the Czech Republic, which facilitated her participation in the Symposium. This work was partially financed by CONICET of Argentina under project PIP 5886/05 and Universidad Nacional de La Plata, under project 11/G072.

\section{References}

Cappa, C., Rubio, M., Martín, M.C., \& McClure-Griffiths, N.M. 2005a, ASPC 344, 179

Cappa, C., Niemela, V., Martín, M.C., \& McClure-Griffiths, N.M. 2005b, A\&A 436, 155

Cutri, R.M. et al. 2003, IPAC, University of Massachusetts

Egan, M.P. et al. 2003, AFRL-VS-TR-2003-1589

Elmegreen, B.G. \& Lada, C.J. 1977, ApJ 214, 725

Martín, M.C., Cappa, C.E. \& Testori, J.C. 2007, in preparation 